\begin{tabular}{|c|l|}
\hline Title & $\begin{array}{l}\text { Changes in the fluctuation of the contraction rhythm of spontaneously beating cardiac my ocytes in cultures with and } \\
\text { without cardiac fibroblasts. }\end{array}$ \\
\hline Author(s) & Hachiro, Takeru; Kawahara, Koichi; Sato, Rie; Y amauchi, Y oshiko; Matsuy ama, Daisuke \\
\hline Citation & $\begin{array}{l}\text { Biosystems, 90/3), 707-715 } \\
\text { https://doi.org/10.1016/.biosystems.2007.02.009 }\end{array}$ \\
\hline Issue Date & $2007-11$ \\
\hline Doc URL & http://hdl.handle.net/2115/32320 \\
\hline Type & article (author version) \\
\hline File Information & BS90-3.pdf \\
\hline
\end{tabular}

Instructions for use 


\title{
Changes in the fluctuation of the contraction rhythm of spontaneously beating cardiac myocytes in cultures with and without cardiac fibroblasts
}

\author{
Takeru Hachiro, Koichi Kawahara, Rie Sato, Yoshiko Yamauchi, and \\ Daisuke Matsuyama
}

Laboratory of Cellular Cybernetics, Graduate School of Information

Science and Technology, Hokkaido University, Sapporo 060-0814, Japan

Address correspondence to:

Koichi Kawahara, PhD

Professor of the Department of Cellular Cybernetics

Graduate School of Information Science and Technology

Hokkaido University

Sapporo 060-0814

Japan

E-mail: kawahara@cellc.ist.hokudai.ac.jp

FAX: +81-11-706-7591 


\section{Summary}

The heart functions as a syncytium of cardiac myocytes and surrounding supportive non-myocytes such as fibroblasts. There is a possibility that a variety of non-myocyte-derived factors affect the maturation of cardiac myocytes in the development of the heart. Cultured neonatal cardiac myocytes contract spontaneously and cyclically. The fluctuation of beating rhythm varies depending on the strength of coupling through gap junctions among cardiac myocytes, indicating that the development of intercellular communication via gap junctions is crucial to the stability of contraction rhythm in cardiac myocytes. In this study, we aimed at elucidating whether and how cardiac fibroblasts affect the development of cardiac myocytes from the point of view of the changes in the fluctuation of the contraction rhythm of cardiac myocytes in cardiac myocyte-fibroblast co-cultures. The present study suggested that cardiac fibroblasts co-cultured with cardiac myocytes enhanced the intercellular communication among myocytes via gap junctions, thereby stabilizing the 
spontaneous contraction rhythm of cultured cardiac myocytes.

\section{Keywords:}

Fluctuation of contraction rhythm; cardiac myocytes; cardiac fibroblasts;

gap junctions; development 


\section{Introduction}

The heart functions as a syncytium of cardiac myocytes and surrounding supportive cells consisting of fibroblasts, endothelial cells, smooth muscle cells, and macrophages (Harada et al., 1997). Although cardiac myocytes make up most of the adult myocardial mass, they make up only $30 \%$ of the total cell number present in the heart; the rest is composed of non-myocytes such as fibroblasts (Eghbali et al., 1988; Zak, 1973). Therefore, there is a possibility that a variety of non-myocyte-derived factors affect the maturation of cardiac myocytes in the development of the heart. In fact, previous studies have revealed that non-myocytes secrete a humoral factor such as endothelin-1 (ET-1) or cardiotrophin-1 (CT-1) regulating the development of cardiac myocytes (Harada et al., 1997; Kuwahara et al., 1999).

Isolated and cultured neonatal cardiac myocytes contract spontaneously and cyclically (Harary and Farley, 1963). The contraction rhythm in the myocytes has the properties of non-linear oscillation, because 
the rhythm is entrained to an externally applied rhythmic electrical stimulation (Glass et al., 1983). In addition, the contraction rhythms of two isolated cardiac myocytes, each of which beats at different frequencies at first, become synchronized after the establishment of mutual contacts, suggesting that mutual entrainment occurs due to electrical and/or mechanical interactions between two myocytes (Jongsma et al., 1987; Kimura et al., 1995; Nakayama et al., 2005; Yamauchi et al., 2002). We have recently demonstrated that the fluctuation of beating rhythm, estimated from the coefficient of variation (CV) of contraction intervals, changes depending on the strength of coupling through gap junctions among spontaneously beating cardiac myocytes (Yamauchi et al., 2002). The reduced fluctuation of beating rhythm depending on the culture period seems to reflect the synchronization of contraction rhythm among myocytes caused by increased mechanical and/or electrical coupling strength via gap junctions. In addition, we have also revealed that treatment of cultured cardiac myocytes with a 12-doxyl stearic acid, a 
reversible blocker of gap junction channels, results in the asynchronization of contraction rhythm among cardiac myocytes and in an increase in the fluctuation of contraction rhythm (Kawahara et al., 2002). Taken together, these findings have led to a suggestion that intercellular communication via gap junction is crucial to the stability of contraction rhythm of cultured cardiac myocytes.

In this study, we aimed at elucidating whether and how cardiac fibroblasts affect the development of cardiac myocytes from the point of view of the changes in the fluctuation of the contraction rhythm of cardiac myocytes in cardiac myocyte-fibroblast co-cultures. The present study suggested that the cardiac fibroblasts enhanced the intercellular communication among myocytes via gap junctions, thereby stabilizing the spontaneous contraction rhythm of cultured cardiac myocytes. 


\section{Materials and methods}

The animal experiments conformed to the "Principles of laboratory animal care” (NIH publication No. 85-23, revised 1996), as well as the "guide for the care and use of laboratory animals", Hokkaido University School of Medicine.

\section{Cell cultures}

The method of culture of cardiac myocytes (CMs) was described elsewhere in detail (Kawahara et al., 2006; Kohashi et al., 2003; Yamauchi et al., 2002). In short, CMs were prepared from ventricles of a few day-old neonatal Wistar rats removed after decapitation. The ventricles were rinsed in a $25 \mathrm{mM}$ HEPES-buffered minimum salt solution (MSS) to remove contaminating blood cell components and then minced with scissors into fragments to be digested with $0.1 \%$ collagenase (Wako Chemical, Tokyo, Japan) in MSS at $37{ }^{\circ} \mathrm{C}$ for $10 \mathrm{~min}$. To separate CMs from cardiac fibroblasts (CFs) based on the selective adhesion technique, 
the cell suspension was poured into 2 or 3 petri dishes ( $\phi 60 \mathrm{~mm}$, Falcon), and incubated in MCDB 107 (containing 5\% FBS, $100 \mathrm{U} / \mathrm{mL}$ penicillin and $100 \mu \mathrm{g} / \mathrm{mL}$ streptomycine) for $60 \mathrm{~min}$ at $37{ }^{\circ} \mathrm{C}$, in $5 \% \mathrm{CO}_{2}$ and $95 \%$ air. By virtue of the procedure, most of the CFs adhere to the dish. After the incubation, the suspension, mostly containing CMs, was collected and centrifuged at $700 \mathrm{rpm}$ for $5 \mathrm{~min}$.

For preparing CF-enriched cultures, the dishes with both CMs and CFs were washed with phosphate-buffered saline (PBS) a few times to completely eliminate CMs and incubated in MCDB107 for 1 to 3 weeks. CFs were seeded at a density of about $1.0 \times 10^{5}$ cells $/ \mathrm{ml}$ in a petri dish coated beforehand with fibronectine. The culture medium was exchanged 12 and 60 hours from seeding. These CF-enriched cultures were also used for the preparation of CM-CF co-cultures.

Following the methods for preparing CM-enriched cultures proposed by Simpson and Savion (1982), the cell suspension after centrifugation was passed through a fine wire mesh screen ( $25 \mu \mathrm{m}$ porosity) to remove 
remaining small aggregates of cells, and finally the isolated CMs remaining were cultured in MCDB 107 supplemented with $100 \mu \mathrm{M}$ 5-bromo-2'-deoxy-uridine (BrdU) to block the proliferation of contaminated CFs at a density of about $2.0 \times 10^{5}$ cells/ml in a petri dish $(\phi 35$ mm, Greiner Bio-One, German) coated beforehand with fibronectine (1.0 $\mu \mathrm{g} / \mathrm{cm}^{2}$, Sigma). The supplementation of BrdU was done for 3 days.

For the preparation of CM-CF co-cultures, CFs obtained as described above were first removed by $0.1 \%$ trypsin-EDTA in PBS after $6 \mathrm{~h}$ of incubation. After stopping enzymatic reactions by adding culture medium containing serum, we centrifuged the cell suspension at $700 \mathrm{rpm}$ for $5 \mathrm{~min}$ twice. The suspension was passed through a fine wire mesh screen (25 $\mu \mathrm{m}$ porosity) and CFs were isolated. The CFs were then seeded at a density of about $1.0 \times 10^{5}$ cells/ml on CM-enriched cultures prepared as described above.

Cultures incubated for 4 or 5 days ( 4 or 5 days in vitro, 4 or 5DIV) were used for the experiments. 


\section{Image analysis}

In this study, the spontaneous contraction rhythm of cultured myocytes was evaluated using a video image recording method. This procedure is described elsewhere in detail (Kawahara et al., 2002; Kohashi et al., 2003; Yamauchi et al., 2002; Yoneyama and Kawahara, 2004). In short, images of beating myocytes were recorded with a CCD camera (WV-BD400, Panasonic, Japan) through a phase-contrast microscope (IX70, OLYMPUS, Japan). A spontaneously beating myocyte was arbitrarily selected from myocytes in the video image. A small area (a square of $25 \times 25$ pixels) of the myocyte where brightness changed considerably with contraction was selected, and the video signals were digitized to an 8-bit number every video frame (30 frames/s) by a video capture board in a personal computer (Power Macintosh 7500/100, Apple).

A reference frame was arbitrarily selected and cross-correlograms were calculated between pixels of the reference frame and those of other 
frames to represent the temporal variation of brightness in the selected area corresponding to the contraction rhythm of the CM.

\section{Immunofluorescence}

The expression of connexin-43 and the sarcomeric $\alpha$-actinin in cultured cells was detected by immunostaining with an anti-connexin-43 rabbit antibody (Sigma, St. Louis, MA) and an anti-sarcomeric- $\alpha$-acitnin mouse antibody (Sigma), respectively. At first, the cells were fixed with 4\% paraformaldehyde for 30 minutes at room temperature. The cells were then incubated with primary antibody over a $24 \mathrm{~h}$ period using a dilution of 1:1000 for both connexin-43 and $\alpha$-actinin. After being washed with PBS, the cells were incubated with a secondary antibody containing 1.0\% goat serum for 30 minutes. For labeling, a 1:100 dilution of FITC-conjugated goat antibody against rabbit IgG (Molecular Probes, Eugene, OR) and mouse IgG (Molecular Probes) was used. The fluorescence markers FITC for rabbit IgG and for mouse IgG were excited 
at $488 \mathrm{~nm}$ and at $531 \mathrm{~nm}$ with incident-light fluorescence microscopy, respectively. Samples were incubated with these secondary antibodies for $3 \mathrm{~h}$ at $37^{\circ} \mathrm{C}$. In addition, the nuclei of cells were stained for $10 \mathrm{~min}$ with hoechest-33342 which were exited at $352 \mathrm{~nm}$.

\section{Chemicals}

Heptanol and carbenoxolone were obtained from Sigma. The other chemicals were purchased from Wako Chem.

\section{Statistical analysis}

The data are expressed as the mean+S.D. Group comparisons were made using one-way ANOVA. Scheffe's test was used to test differences of a pair of data points and a $P$ value of less than 0.05 was considered significant. 


\section{Results}

To investigate the interactions between cardiac myocytes and fibroblasts during the development of cultured cardiac myocytes, we first established three kinds of cultures; that is, cardiac myocyte-enriched (CM) cultures, co-cultures of cardiac myocytes and fibroblasts (CM-CF), and cardiac fibroblast-enriched cultures (CF). An immunocytochemical analysis using an anti- $\alpha$-actinin antibody has revealed that most of the cultured cells were stained with the antibody in CM cultures (Fig. 1B and 1F). The sarcomeric $\alpha$-actinin is specifically expressed in cardiac myocytes (Sucharov et al., 2006; Zolk et al., 2004). The nuclei of cells were counterstained with Hoechest-33342. In contrast, many cells not stained with the anti- $\alpha$-actinin antibody were identified in CM-CF co-cultures (Fig. 1D and 1H), indicating that the cultures were mixed. No cells were stained with the antibody in CF cultures (Fig. 1J). There were no distinct morphological differences in cardiac myocytes between cells in the CM cultures and CM-CF co-cultures. 
We have recently demonstrated that the fluctuation of beating rhythm changes depending on the developmental stage of cultured cardiac myocytes (Yamauchi et al., 2002). The developmental changes in the fluctuation of beating rhythm seem to reflect the changes in mechanical and/or electrical coupling strength via gap junctions among cardiac myocytes. Therefore, we next investigated whether there exists differences in the fluctuation of beating rhythm between myocytes in CM cultures and CM-CF co-cultures. On day 4 in vitro (4 DIV), the contraction rhythm in almost all the cardiac myocytes in both CM cultures and CM-CF co-cultures was synchronized with each other (Nakayama et al., 2005). Figure 2 shows the contraction rhythm of cardiac myocytes in CM cultures (Fig. 2A) and in CM-CF co-cultures (Fig. 2B). There was no significant difference in the beating intervals of myocytes between CM cultures and CM-CF co-cultures (Fig. 2C). However, the fluctuation of beating rhythm, estimated from the co-efficient of variation (CV) of the contraction intervals, in cardiac myocytes in CM cultures was significantly 
greater than that in CM-CF co-cultures (Fig. 2D), suggesting that co-culturing cardiac fibroblasts with cardiac myocytes made the contraction rhythm of cardiac myocytes stable. Our previous study has suggested that the developmental changes in the fluctuation of beating rhythm seem to reflect the changes in mechanical and/or electrical coupling strength via gap junctions among cardiac myocytes. Therefore, we next investigated whether there was a difference in the expression and/or distribution of gap junctional proteins between myocytes in CM cultures and in CM-CF co-cultures.

In cultured rat neonatal cardiac myocytes, connexin-43 (Cx43) is a primary subtype constituting gap junction channels (Dhein et al., 2000), although other connexin subtypes (Cx45, Cx43, Cx40, and Cx37) are also expressed (Garcia-Dorado and Ruiz-Meana, 2000). Thus, in this study, an immunocytochemical analysis using an anti-Cx43 antibody was performed to investigate whether the gap junctional intercellular coupling between cardiac myocytes changed depending on the culture conditions. Although 
the expression of $\mathrm{Cx} 43$ was observed as a line or punctuated spots in the intercellular space both in CM cultures and CM-CF co-cultures, it seemed to be more abundant in the co-cultures (Figs. 3B and D). It should be noted that the fluorescence signal from $\mathrm{Cx} 43$ was identified either at the border of two adjacent cardiac myocytes or in the cytoplasm of myocytes (Figs. 3F and $\mathrm{H}$ ), but not in other border zones such as the boundary between either myocytes and fibroblasts or fibroblasts and fibroblasts. Little Cx43 expression was observed in cardiac fibroblast-enriched (CF) cultures (Fig. 3J). These results suggested that cardiac fibroblasts increased the development of gap junctional intercellular communication among cardiac myocytes in CM-CF co-cultures.

The above findings raised the possibility that the decreased fluctuation in the contraction rhythm of cardiac myocytes in CM-CF co-cultures (Fig. 2) was due to the increased gap junctional communication among the myocytes. If this was the case, pharmacological blockade of gap junctional communication in CM-CF co-cultures would result in an 
increase in the fluctuation of contraction rhythm of cardiac myocytes. We therefore examined whether treatment of CM-CF co-cultures with blockers of gap junction channels resulted in an increase in the fluctuation of spontaneous contraction rhythm. Treatment with $50 \mu \mathrm{M}$ carbenoxolone, a water-soluble derivative of 18-alpha-glycyrrhetinic acid, a blocker of gap junctional cellular communication (Gittens et al., 2002; Tong et al., 2006), resulted in a significant reversible increase in the co-efficient of variation of beating intervals (Fig. 4E). In addition, the treatment significantly elongated the beating intervals (Fig. 4D). Treatment with $500 \mu \mathrm{M}$ heptanol, a well-known blocker of gap junction channels, also produced a significant reversible increase in the fluctuation of contraction rhythm (Fig. 5). Treatment with $300 \mu \mathrm{M}$ heptanol increased the fluctuation as well (data not shown). 


\section{Discussion}

The present study has demonstrated that cardiac fibroblasts co-cultured with cardiac myocytes possibly enhanced the intercellular communication among myocytes via gap junctions, thereby stabilizing the spontaneous contraction rhythm of the myocytes.

In this study, we established three kinds of cultures; that is, cardiac myocyte-enriched cultures (CM), co-cultures of cardiac myocytes and fibroblasts (CM-CF), and cardiac fibroblast-enriched cultures (CF). During the isolation of heart cells, however, it is possible that non-myocytes other than cardiac fibroblasts contaminated the CM-CF co-cultures and CF cultures. A recent study has revealed that the expression of Discoidin Domain Receptor 2 (DDR2), one of the receptor tyrosine kinase (RTK) families, is restricted to the cardiac fibroblasts in the heart (Moraris et al., 2005). In that study, the cardiac fibroblasts isolated from the neonatal rat heart expressed DDR2. Therefore, we examined whether the isolated cardiac fibroblast-like cells in our cultures expressed 
DDR2. An immunocytochemical analysis using anti-DDR2 antibody revealed that the isolated cardiac fibroblast-like cells expressed DDR2 (data not shown). This result suggested that the fibroblast-like non-myocytes in our cultures were cardiac fibroblasts.

We have recently demonstrated that the fluctuation of beating rhythm, estimated from the coefficient of variation (CV) of contraction intervals, changes depending on the strength of coupling through gap junctions (GJ) among spontaneously beating cardiac myocytes (Yamauchi et al., 2002). The reduced fluctuation of the beating rhythm of cardiac myocytes in CM-CF co-cultures (Fig. 2D) seems to reflect the increase in mechanical and/or electrical coupling strength via GJs among cardiac myocytes. Thus, we have examined in the present study whether the expression of $\mathrm{Cx} 43$, a primary protein constituting GJs in cardiac myocytes (Dhein et al., 2000), varied depending on culture conditions. An immunocytochemical analysis using an anti-Cx43 antibody has revealed that the $\mathrm{Cx} 43$ seemed more abundant in the cardiac myocytes in CM-CF co-cultures than those in 
CM cultures (Fig. 3). However, a preliminary Western blot analysis by us showed that there was not a statistically significant difference between the CM-CF and CM cultures in the quantity of Cx43 protein (data not shown). We have previously reported that the gap junctional communication among cells was not fully developed in cardiac myocytes in the young cultures seeded at relatively low density (Nakayama et al., in press). In such cultures, a dye transfer analysis by microinjecting the lucifer yellow dye into a single myocyte also supported our inference that gap junction channels are not fully developed, although the expression of Cx43 protein was clearly identified (Nakayama et al., in press). Therefore, the difference in the fluctuation of beating intervals between myocytes in the CM-CF and CM cultures possibly originates from differences in the development of functional GJs among cardiac myocytes.

The present study mainly focused on the interactions between CM and CF. That is, we here investigated whether CF co-cultured with CM changed the development of the functional characteristics of cardiac 
myocytes. In fact, previous studies have revealed that non-myocytes secrete a humoral factor such as endothelin-1 (ET-1) or cardiotrophin-1 (CT-1) regulating the development of cardiac myocytes (Harada et al., 1997; Kuwahara et al., 1999). However, there is a possibility that MC-MC interactions are also critical to the development of MC themselves. In the present study, the density of CM in CM cultures was the same as that in CM-CF co-cultures, suggesting that the CM-CM interactions did not primarily contribute to the difference in the development of gap junctional communication among CM depending on culture conditions observed in this study. However, the possibility that CF would change the CM-CM interactions and affect the development of CM cannot be excluded. That is, CF would indirectly affect the development of CM via CF-induced changes in the CM-CM interactions.

We have recently demonstrated that the extracellular ATP-purinoceptor system is responsible for the intercellular synchronization of intracellular $\mathrm{Ca}^{2+}$ oscillation among cardiac myocytes 
(Nakayama et al., in press). The dynamics of intracellular free $\mathrm{Ca}^{2+}$ directly affects the spontaneous rhythmic contraction of cardiac myocytes. Thus, there is a possibility that ATP released from cardiac myocytes via connexin hemichannels (Contreras et al., 2002; Stout et al., 2002) or maxi-anion channels (Dutta et al., 2004) would affect the fluctuation of spontaneous contraction rhythm of cardiac myocytes. Further studies will be needed to clarify this issue. 


\section{Acknowledgments}

This work was supported by a grant-in-aid for scientific research from the Ministry of Education, Science, and Culture of Japan (16300145) to KK. 


\section{References}

Contreras, J.E., Sánchez, H.A., Eugenin, E.A., Speidel, D., Theis, M., Willecke, K., Bukauskas, F.F., Bennett, V.L., Sáez, J.C., 2002. Metabolic inhibition induces opening of unapposed connexin 43 gap junction hemichannels and reduces gap junctional communication in cortical astrocytes in culture. Proc. Natl. Acad. Sci. USA 99, 495-500.

Dhein, S., Pejman, P., Krüsemann, K., 2000. Effects of $I_{\text {K.ATP }}$ blockers glibenclamide and HMR1883 on cardiac electrophysiology during ischemia and reperfusion. Europ. J. Pharmacol. 398, 273-284.

Dutta, A.K., Sabirov, R.Z., Uramoto, H., Okada, Y., 2004. Role of ATP-conductive anion channel in ATP release from neonatal rat cardiomyocytes in ischemic or hypoxic conditions. J. Physiol. 559.3, 799-812.

Eghbali, M., Czaja, M.J., Zeydel, M., Weiner, F.R., Zern, M.A., Seifter, S., Blumenfeld, O.O., 1988. Collagen chain mRNAs in isolated heart cells from young and adult rats. J. Mol. Cell. 20, 267-276.

Garcia-Dorado, D., Ruiz-Meana, M., 2000. Propagation of cell death during myocardial reperfusion. News Physiol. Sci. 15, 326-330.

Gittens, J.E.I., Mhawi, A.A., Lidington, D., Ouellette, Y., Kidder, G.M., 2003. Functional analysis of gap junctions in ovarian granulosa cells: distinct role for connexin43 in early stages of folliculogenesis. Am. J. Physiol. Cell. Physil. 284, C880-C887.

Glass, L., Guevara, M.R., Shrier, A., Perez, R., 1983. Bifurcation and chaos in a periodically stimulated cardiac oscillator. Physica D 7, 89-101. 
Harada, M., Itoh, H., Nakagawa, O., Ogawa, Y., Miyamoto, Y., Kuwahara, K., Ogawa, E., Igaki, T., Yamashita, J., Masuda, I., Yoshimasa, T., Tanaka, I., Saito, Y., Nakao, K., 1997. Significance of ventricular myocytes and nonmyocytes interaction during cardiocyte hypertrophy. Circulation 96, 3737-3744.

Harary, I., Farley, B., 1963. In vitro studies on single beating rat heart cells; I. Growth and organization. Exp. Cell Res. 29, 451-465.

Jongsma, H.J., Masson-Pevet, M., Tsjernina, L., 1987. The development of beat-rate synchronization of rat myocyte pairs in cell culture. Basic Res. Cardiol. 82, 454-464.

Kawahara, K., Abe, R., Yamauchi, Y., Kohashi, M., 2002. Fluctuations in contraction rhythm during simulated ischemia/reperfusion in cultured cardiac myocytes from neonatal rats. Biol. Rhythm Res. 33, 339-350.

Kawahara, K., Hachiro, T., Yokokawa, T., Nakajima, T., Nakayama, Y., 2006. Ischemia/reperfusion-induced death of cardiac myocytes: possible involvement of nitric oxide in the coordination of ATP supply and demand during ischemia. J. Mol. Cell. Cardiol. 40, 35-46.

Kimura, H., Oyamada, Y., Ohshika, H., Mori, M., Oyamada, M., 1995. Reversible inhibition of gap junctional intercellular communication, synchronous contraction, and synchronism of intracellular $\mathrm{Ca}^{2+}$ fluctuation in cultured neonatal rat cardiac myocytes by heptanol. Exp. Cell Res. 220, 348-356.

Kohashi, M., Kawahara, K., Yamauchi, Y., 2003. Carbachol-induced suppression of contraction rhythm in spontane ously beating cultured cardiac myocytes from neonatal rats. Biol. Rhythm Res. 34, 367-381. 
Kuwahara, K., Saito, Y., Harada, M., Ishikawa, M., Ogawa, E., Miyamoto, Y., Hamanaka, I., Kamitani, S., Kajiyama, N., Takahashi, N., Nakagawa, O., Masuda, I., Nakao, K., 1999. Involvement of cardiotrophin-1 in cardiac myocyte-nonmyocyte interactions during hypertrophy of rat cardiac myocytes in vitro. Circulation, 100, 1116-1124.

Morales, M.O., Price, R.L., Goldsmith, E.C., 2005. Expression of Discoidin Domain Receptor 2 (DDR2) in the developing heart. Microsc. Microanal. 11, 260-267.

Nakayama, Y., Kawahara, K., Hachiro, T., Yamauchi, Y., Yoneyama, M., 2006. Possible involvement of ATP-purinoceptor signaling in the intercellular synchronization of intracellular $\mathrm{Ca}^{2+}$ oscillation in cultured cardiac myocytes. BioSystems, in press.

Nakayama, Y., Kawahara, K., Yoneyama, M., Hachiro, T., 2005 b. Rhythmic contraction and intracellular $\mathrm{Ca}^{2+}$ oscillatory rhythm in spontaneously beating cultured cardiac myocytes. Biol. Rhythm Res. 36, 317-326.

Simpson, P., Savion, S., 1982. Differentiation of rat myocytes in single cell cultures with and without proliferating nonmyocardial cells: Cross-striations and ultrastructure, and chronotropic response to isoproterenol. Circ. Res. 50, 101-116.

Stout, C.E., Costantin, J.L., Naus, C.C.G., Charles, A.C., 2002. Intercellular calcium signaling in astrocytes via ATP release through connexin hemichannels. J. Biol. Chem. 277, 10482-10488.

Sucharov, C.C., Mariner, P.D., Nunley, K.R., Long, C., Leinwand, L., Bristow, M.R., 2006. A $\beta 1$-adrenergic receptor CAM kinase II-dependent pathway mediates cardiac myocyte fetal gene induction. 
Am. J. Physiol. 291, H1299-H1308.

Tong, D., Gittens, J.E.I., Kidder, G.M., Bai, D., 2006. Patch-clamp study reveals that the importance of connexin43-mediated gap junctional communication for folliculogenesis is strain specific in the mouse. Am. J. Physiol. Cell. Physil. 290, C290-C297.

Yamauchi, Y., Harada, A., Kawahara, K., 2002. Changes in the fluctuation of interbeat intervals in spontaneously beating cultured cardiac myocytes: experimental and simulation studies. Biol. Cybern. 86, 147-154.

Yoneyama, M., Kawahara, K., 2004. Fluctuation dynamics in coupled oscillator systems of spontaneously beating cultured cardiac myocytes. Physical Rev. E. 70, 21904.

Zak, R., 1973. Cell proliferation during cardiac growth. Am. J. Cardiol. 31, 211-219.

Zolk, O., Münzel, F., Eschenhagen, T., 2004. Effects of chronic endothelin-1 stimulation on cardiac myocyte contractile function. Am. J. Physiol. 286, H1248-H1257. 


\section{Figure captions}

Fig. 1

Cardiac myocyte-enriched (CM), cardiac fibroblast-enriched (CF), and cardiac myocyte-fibroblast (CM-CF) co-cultures. An immunocytochemical analysis of 4 DIV cultures using an anti-actinin antibody to identify the cardiac myocytes revealed that most of the cells in

CM cultures were actinin-positive cardiac myocytes (B and F; red fluorescence), but actinin-positive cells were not detected in CF cultures (J). The nuclei of cells were stained with Hoechst 33342 (B, D, F, H, and J; blue fluorescence). In CM-CF co-cultures, many actinin-negative cells were identified together with the actinin-positive cardiac myocytes, indicating that the cultures were mixed. Figures A, C, E, G, and I show phase-contrast images of the cultures. The scale bars indicate $100 \mu \mathrm{m}$.

Fig. 2 
Fluctuation of spontaneous contraction rhythm in cardiac myocytes in $\mathrm{CM}$ and $\mathrm{CM}-\mathrm{CF}$ co-cultures. Figures $\mathrm{A}$ and $\mathrm{B}$ show the contraction rhythm of cardiac myocytes in CM and CM-CF co-cultures, respectively. Figure $\mathrm{C}$ shows the average beating intervals of cardiac myocytes in both CM and CM-CF co-cultures (10 cells in 4 different CM or CM-CF co-cultures). Figure D illustrates the coefficient of variation (CV) of beating intervals of cardiac myocytes in both CM and CM-CF co-cultures (10 cells in 4 different CM or CM-CF co-cultures). It should be noted that there was no significant difference in the mean beating intervals, but the $\mathrm{CV}$ of beating intervals of cardiac myocytes in CM-CF co-cultures was significantly smaller than that in CM cultures. Data are expressed as the mean + SD. $\quad * \mathrm{p}<0.05$

Fig. 3 
Expression and distribution of gap junctional protein (Cx43) in CM and CM-CF co-cultures. An immunocytochemical analysis of 4 DIV cultures using an anti-Cx43 antibody to identify the expression and distribution of gap junction proteins in cultured cardiac myocytes in CM (B and F) and CM-CF co-cultures ( $\mathrm{D}$ and $\mathrm{H}$ ) revealed that $\mathrm{Cx} 43$ protein (green fluorescence) seemed to be more abundantly expressed in CM-CF co-cultures (D) than CM cultures (B), but both kinds of cultures had a dot-like $\mathrm{Cx} 43$ distribution linking adjacent cells ( $\mathrm{F}$ and $\mathrm{H})$. In contrast, little Cx43 protein was observed in CF cultures (J). Figures A, C, E, G, and I show phase-contrast images of the cultures. The scale bars indicate $100 \mu \mathrm{m}$.

Fig. 4

A blockade of gap junctional intercellular communication with carbenoxolone resulted in a reversible increase in the coefficient of 
variation $(\mathrm{CV})$ of beating intervals. Figures $\mathrm{A}, \mathrm{B}$, and $\mathrm{C}$ show the contraction rhythm of myocytes before the treatment (A), 20 min after the start of carbenoxolone (50 $\mu \mathrm{M})$ treatment (B), and 20 min (C) after the washout, respectively. Figure D shows the carbenoxolone-induced reversible changes in the mean beating intervals (10 cells in 4 different CM-CF co-cultures). Figure F illustrates the carbenoxolone-induced reversible changes in the CV of beating intervals (10 cells in 4 different CM-CF co-cultures). Data are expressed as the mean $+\mathrm{SD} . \quad * \mathrm{p}<0.05$.

Fig. 5

A blockade of gap junctional intercellular communication with heptanol resulted in a reversible increase in the coefficient of variation (CV) of beating intervals. Figures A, B, and C show the contraction rhythm of myocytes before the treatment (A), 20 min after the start of heptanol (500 $\mu \mathrm{M}$ ) treatment (B), and 20 min (C) after the washout, respectively. Figure 
D shows the heptanol-induced reversible changes in the mean beating intervals (10 cells in 4 different CM-CF co-cultures). Figure F illustrates the heptanol-induced reversible changes in the CV of beating intervals (10 cells in 4 different CM-CF co-cultures). Data are expressed as the mean + SD. $\quad * \mathrm{p}<0.05$ 
A

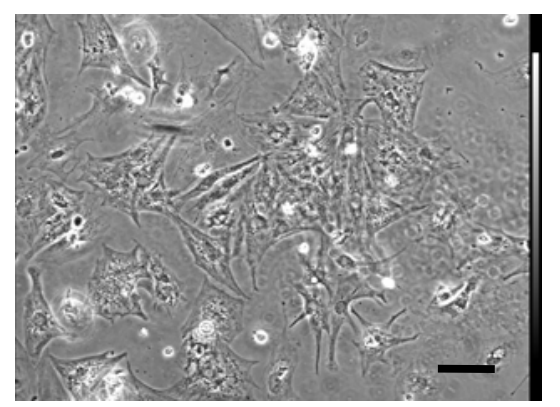

B
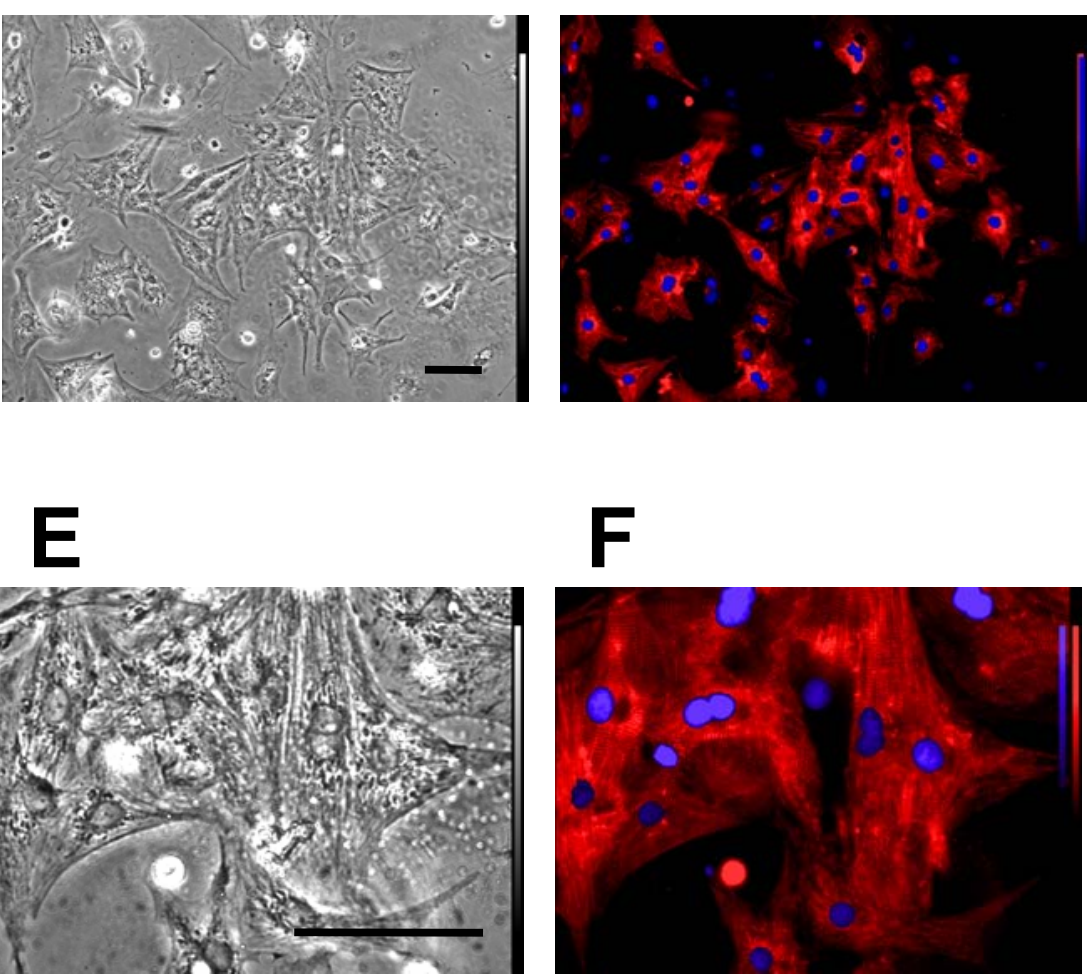

F

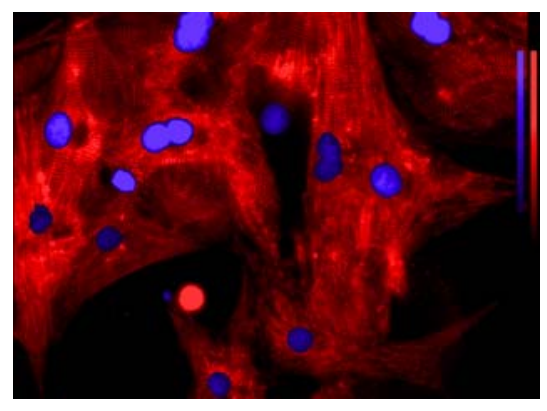

I

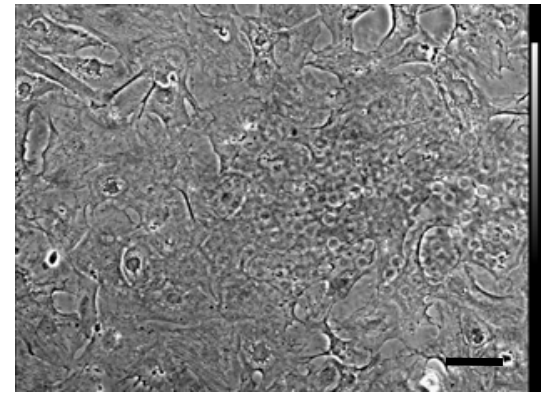

C

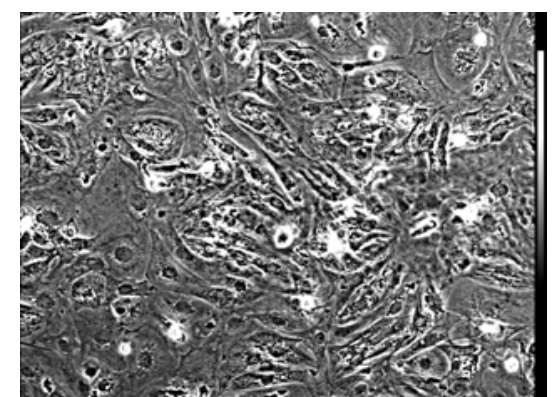

G

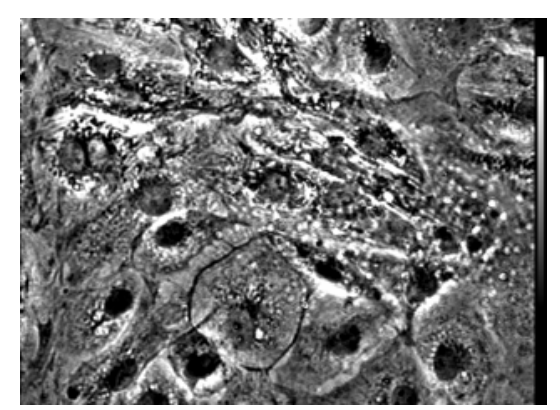

J

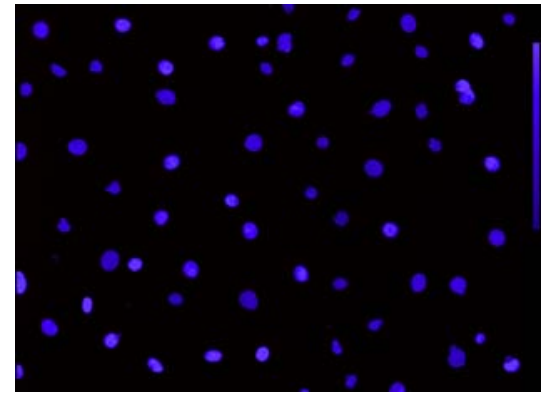

D

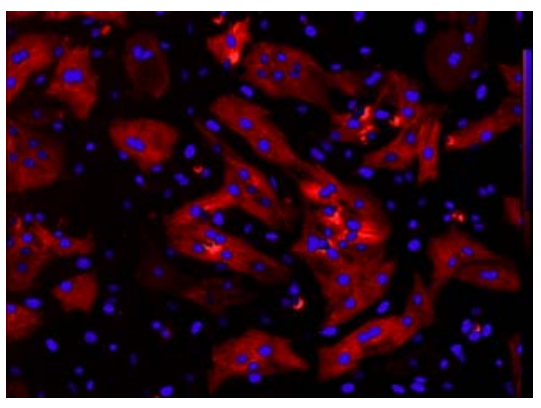

H

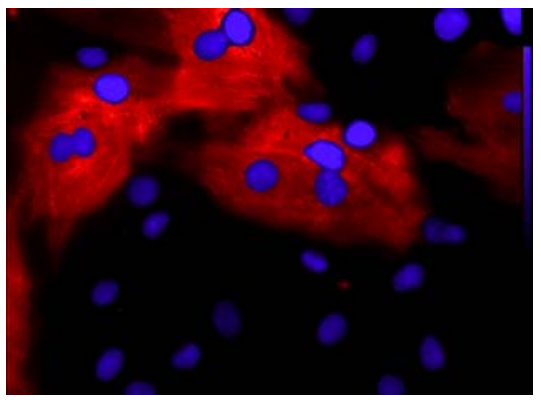


A

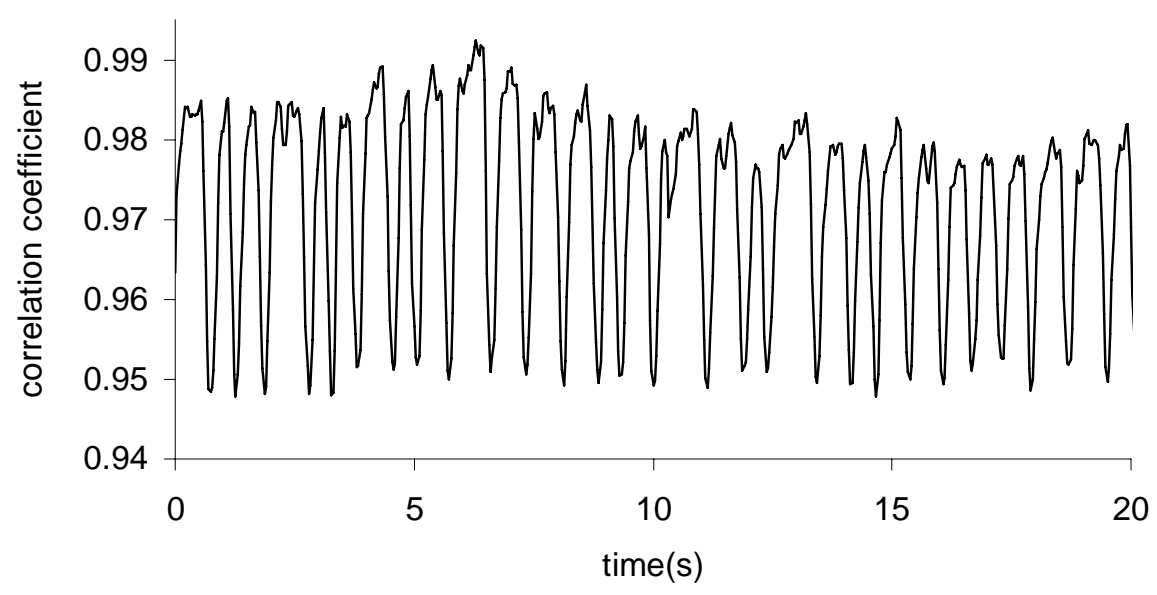

\section{B}

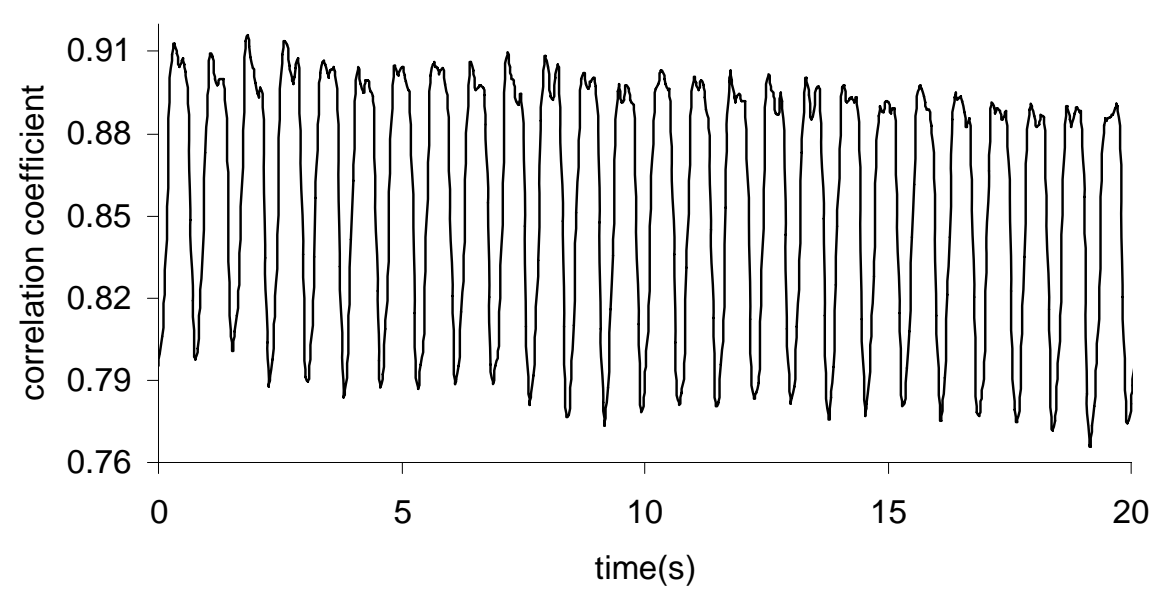

C

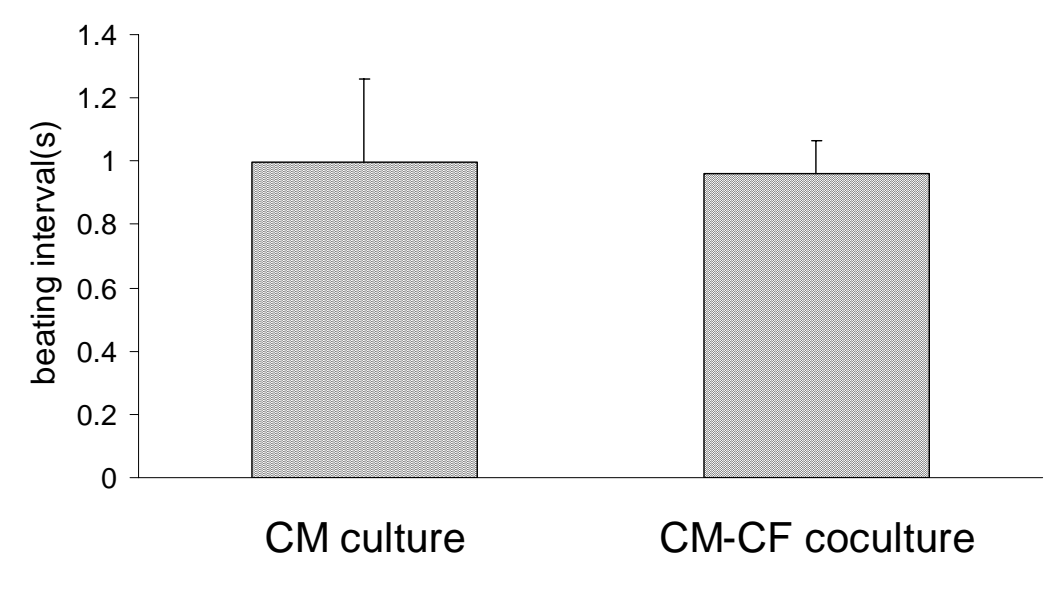

D

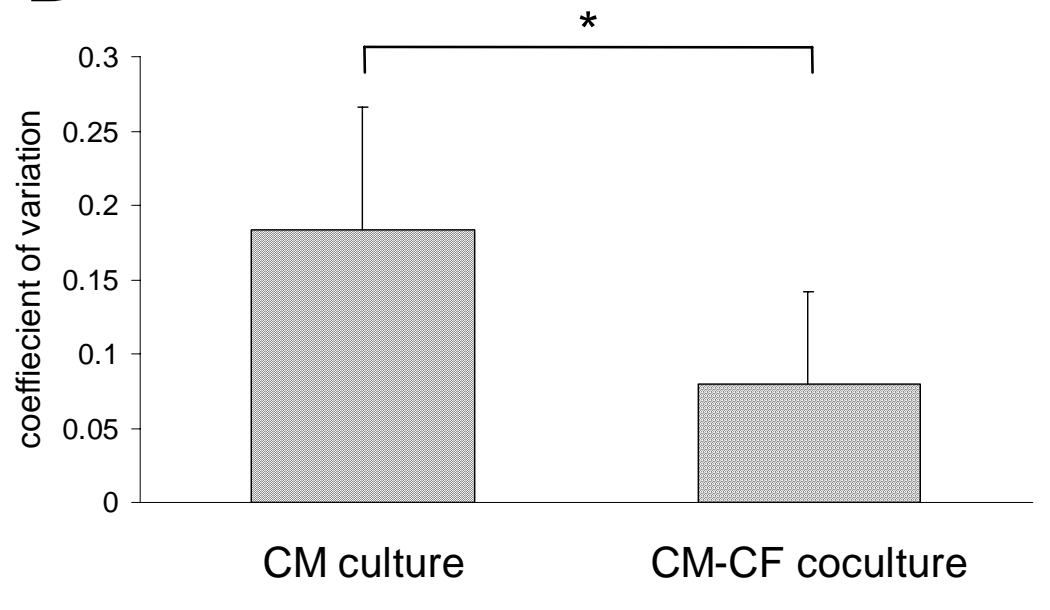


A $B$
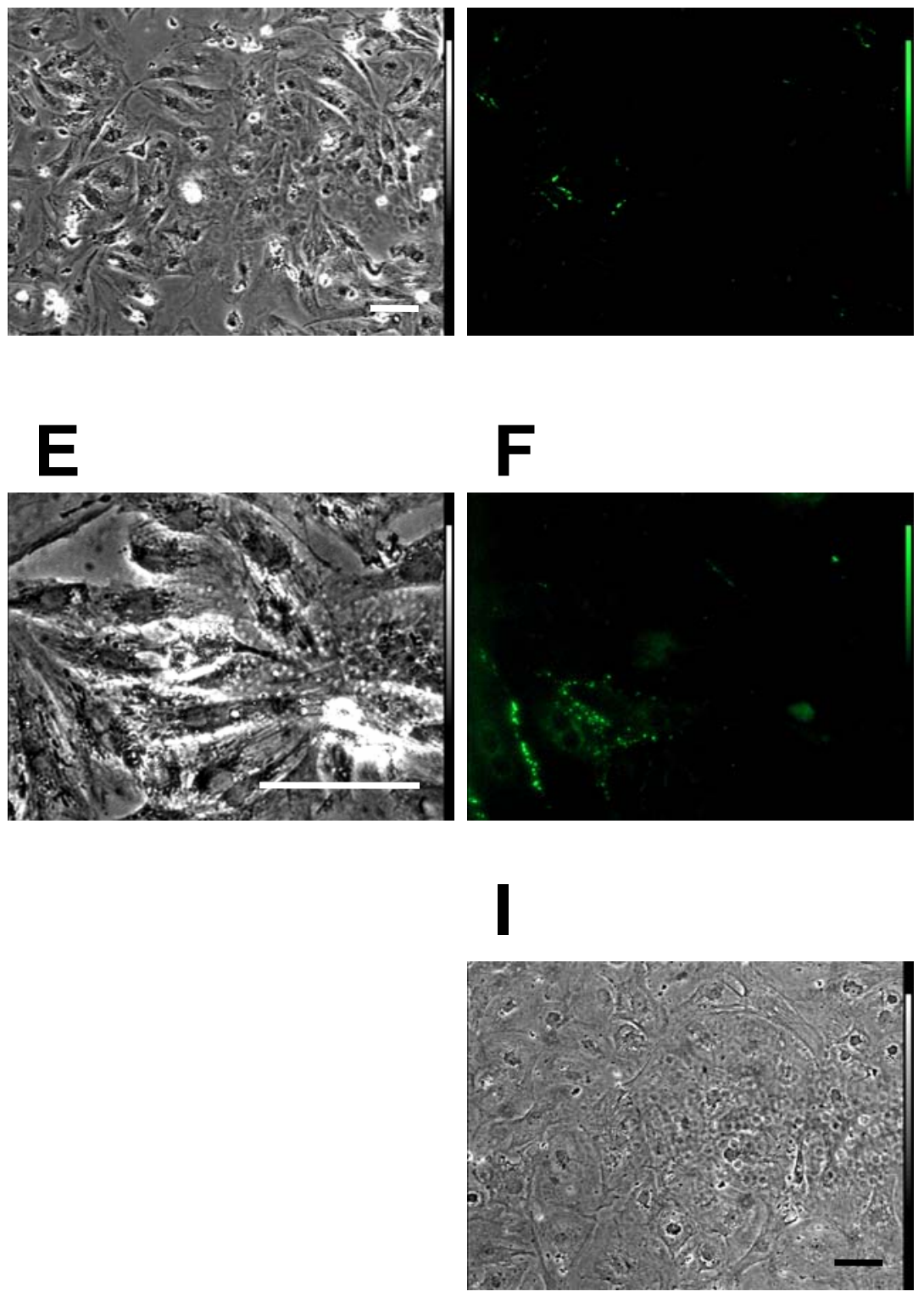

C

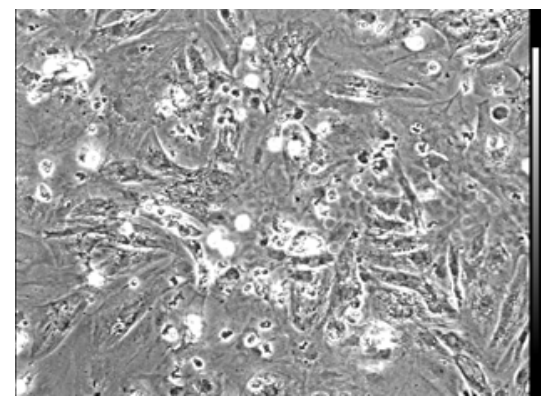

G

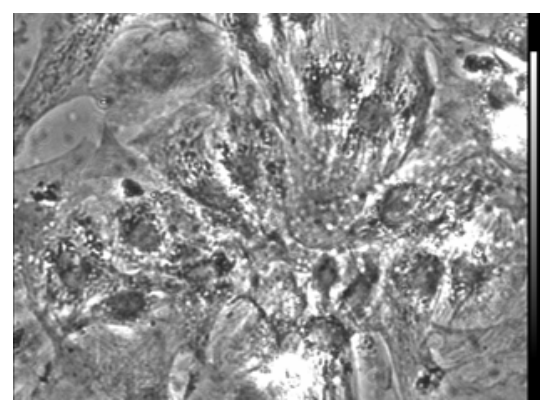

J

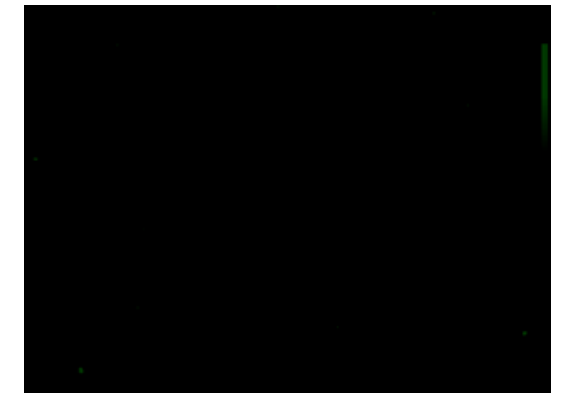

D

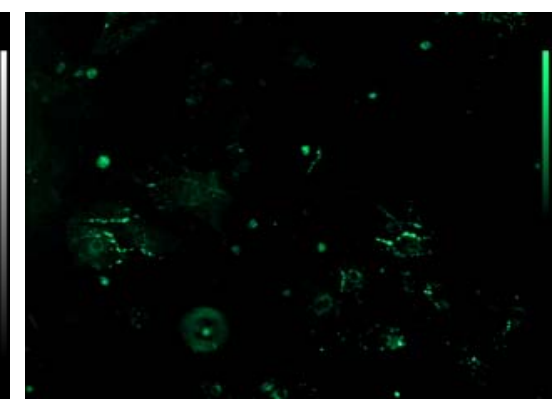

H

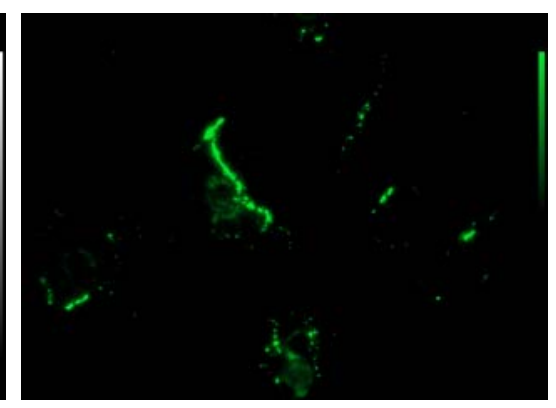


A

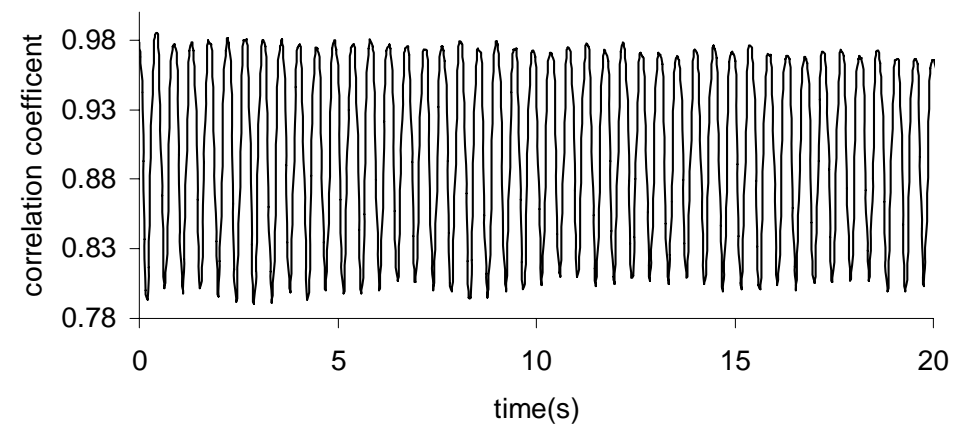

B

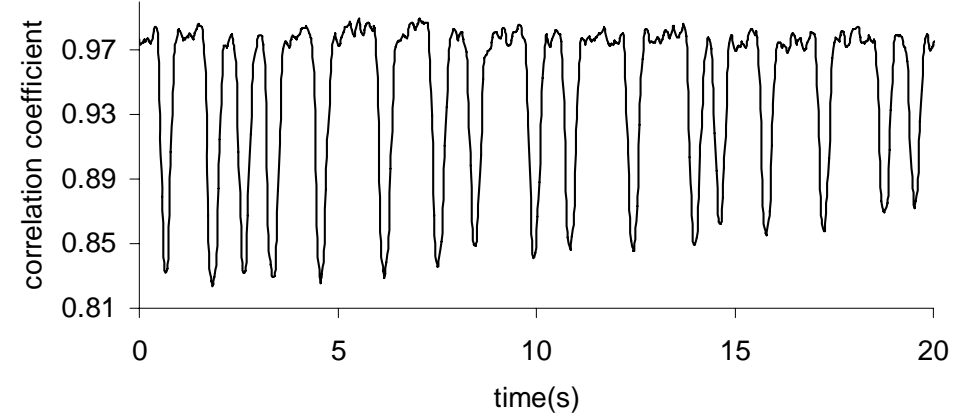

C

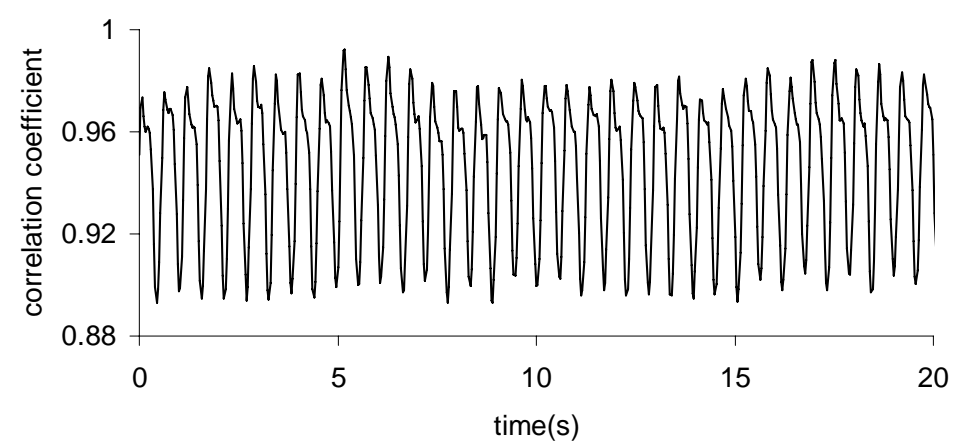

D

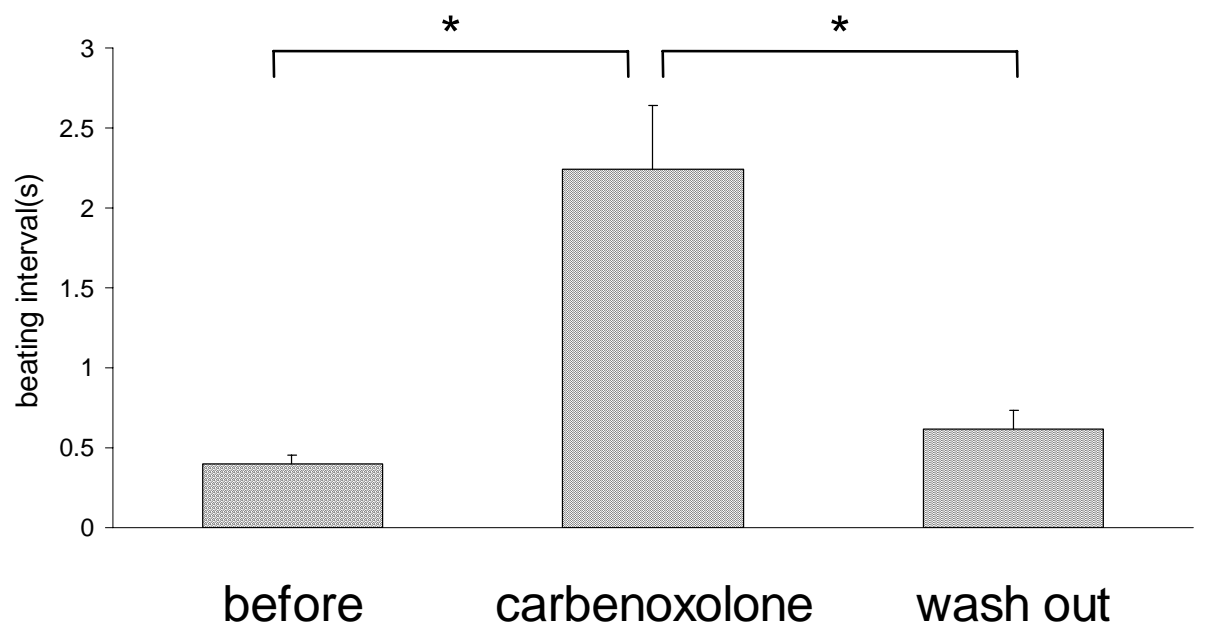

E

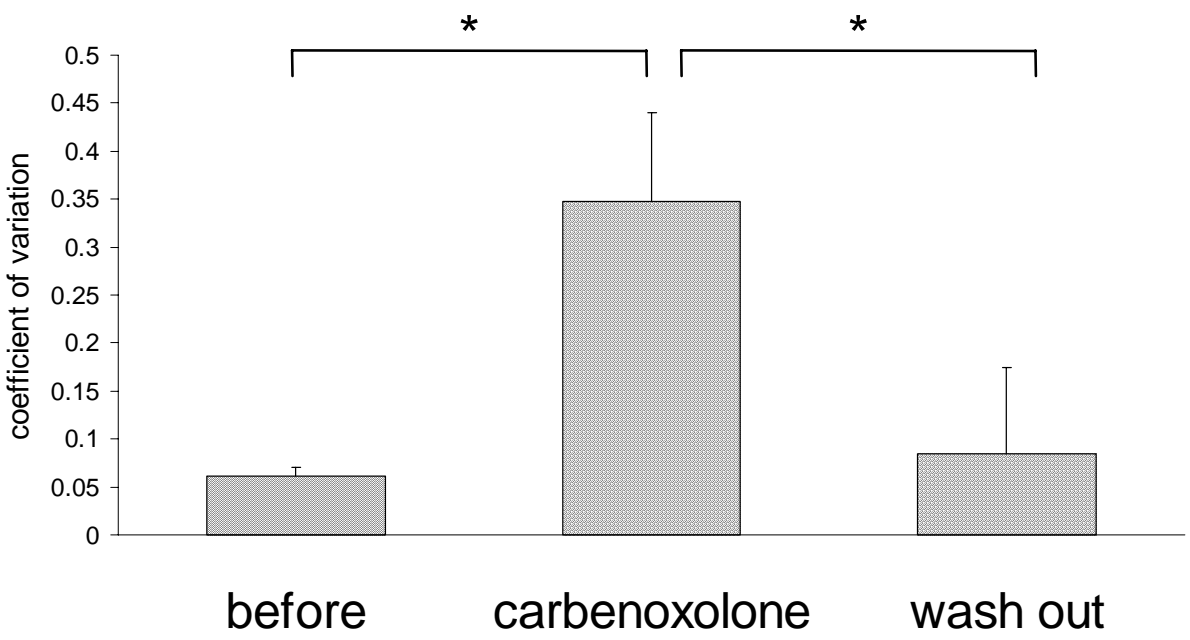


A

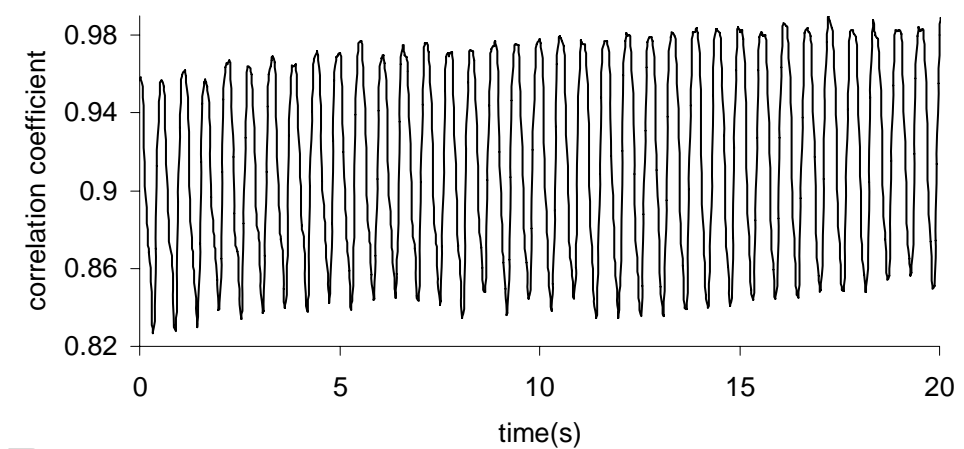

B

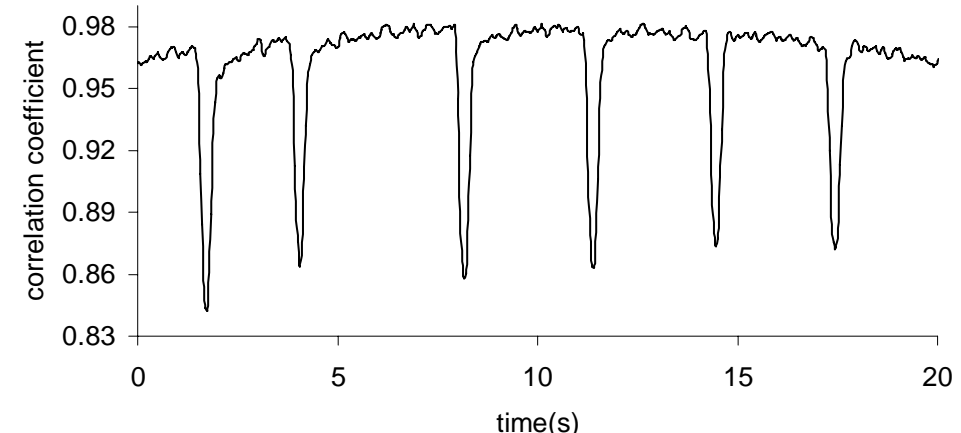

C

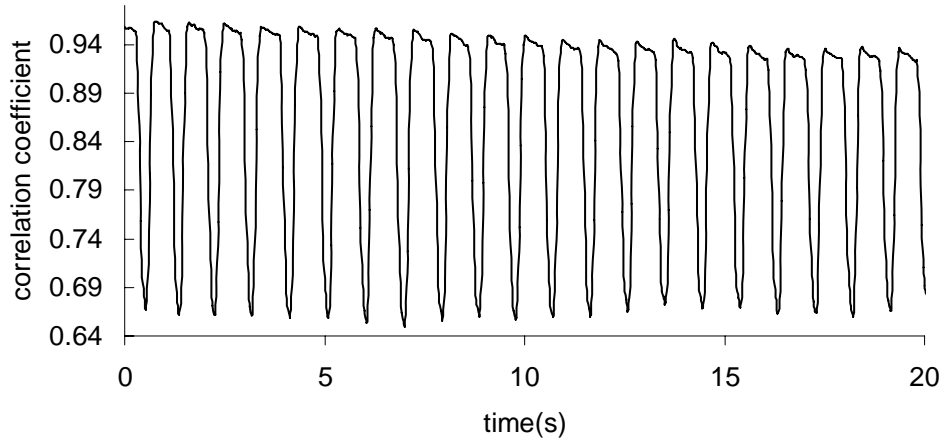

D

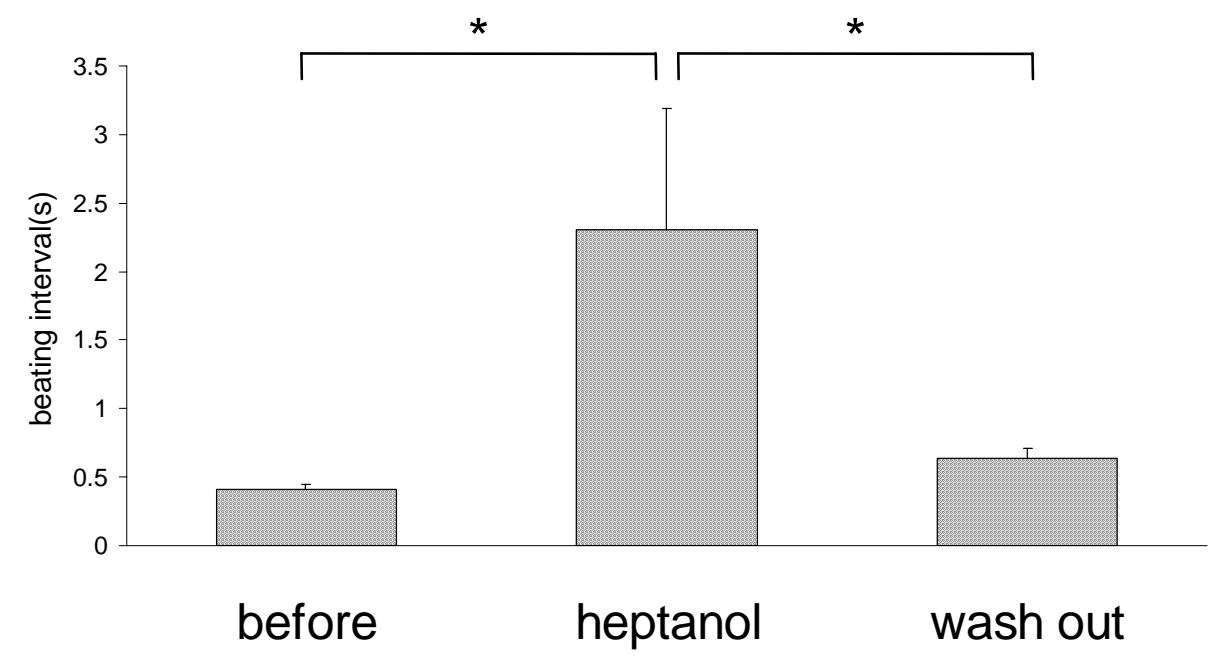

E

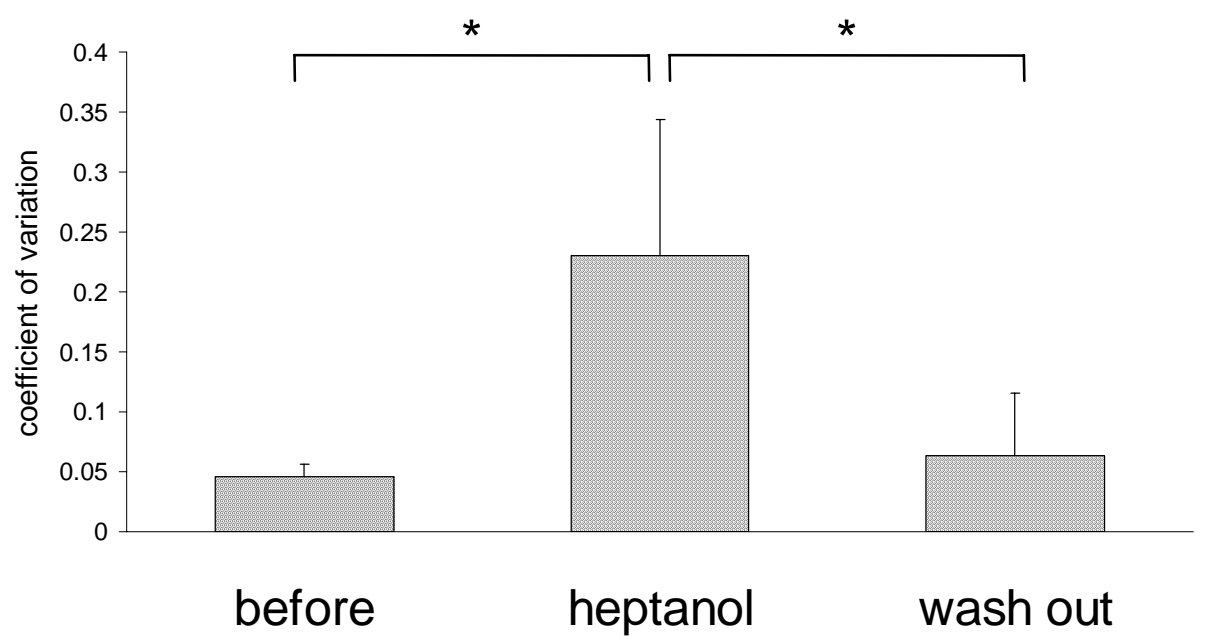

